\title{
Dynamic modelling of consumers' inconvenience associated with demand flexibility potentials
}

\author{
Evelyn Heylen*, Dimitrios Papadaskalopoulos ${ }^{\bullet}$, Ioannis Konstantelos ${ }^{\bullet}$, Goran Strbac ${ }^{\bullet}$ \\ ${ }^{*} K U$ Leuven - EnergyVille, Department of Electrical Engineering \\ Kasteelpark Arenberg 10 PB 2445, 3001 Leuven-Heverlee, Belgium \\ - Imperial College London, Department of Electrical Engineering, Control and Power research group, \\ South Kensington Campus, London, UK
}

\begin{abstract}
Demand flexibility, involving the potential to reduce or temporally defer electricity demand, is regarded as a key enabler for transitioning to a secure, cost-efficient and low-carbon energy future. However, previous work has not comprehensively modelled the inconvenience experienced by end-consumers due to demand modifications, since it has focused on static modeling approaches. This paper presents a novel model of inconvenience cost that simultaneously accounts for differentiated preferences of consumer groups, time and duration of interruptions, differentiated valuation of different units of power and temporal redistribution of shiftable loads. This model is dynamic and future-agnostic, implying that it captures the time-coupling characteristics of consumers' flexibility and the temporal evolution of interruptions, without resorting to the unrealistic assumption that time and duration of interruptions are foreknown. The model is quantitatively informed by publicly available surveys combined with realistic assumptions and suitable sensitivity analyses regarding aspects excluded from existing surveys. In the examined case studies, the developed model is applied to manage an aggregator's portfolio in a scenario involving emergence of an adequacy issue in the Belgian system. The results illustrate how considering each of the above factors affects demand management decisions and the inconvenience cost, revealing the value of the developed model.
\end{abstract}

Keywords: Inconvenience cost, interruption cost, value of lost load, power system service reliability, demand-side flexibility

\section{Nomenclature}

\section{Indices and Sets}

$l \quad$ Index of loads

\footnotetext{
${ }^{*}$ Corresponding author

Email address: evelyn.heylen@esat.kuleuven.be (Evelyn Heylen*)
} 
$t \quad$ Index of time periods

$T_{l}^{\text {shift }}$ Set of time periods when shiftable load $l$ can be activated

$\mathcal{L}^{\text {curt }} \quad$ Set of curtailable loads

$\mathcal{L}^{\text {shift }}$ Set of shiftable loads

\section{Control variables}

$P_{l, t}^{c u r t}$ Curtailed power of curtailable load $l$ in time period $t$

$z_{l, t} \quad$ Binary variable indicating whether the shiftable load $l$ is activated in time period $t$ ( 1 if it is, 0 if it is not)

\section{State variables}

$D_{l, t} \quad$ Auxiliary variable representing the interruption duration (if $D_{l, t}>0$ ) or the time since the last interruption (if $D_{l, t}<0$ ) for load $l$ at time $t$

$D_{l, t}^{i n t} \quad$ Interruption duration for load $l$ at time $t$

$D_{l, t}^{\text {int,shift }}$ Interruption duration of shiftable load $l$ at time $t$

$D_{l, t}^{\text {norm }}$ Time since last interruption for load $l$ at time $t$

$Q_{l, t}^{\text {shift }}$ Binary variable of late activation of shiftable load $l$ in time period $t$ ( 1 if $t$ is beyond the acceptable activation time frame and the load is activated, 0 if $t$ is within the acceptable activation time frame or $t$ is beyond the acceptable activation time frame and the load is not activated.)

$Y_{l, t} \quad$ Binary variable indicating the connection status of load $l$ in time period $t$ ( 1 if the baseline demand of curtailable load $l$ is supplied or shiftable load $l$ is activated, 0 if (part of) curtailable load $l$ is not supplied or shiftable load $l$ is not activated)

$Z_{l, t} \quad$ Auxiliary binary variable indicating whether shiftable load $l$ has been activated by time $t$ (1 if it has been activated, 0 if it has not been activated)

$\delta_{l, t}^{\text {shift }}$ Activation delay of shiftable load $l$ at time $t$

\section{Output variables}

$C_{l, t}^{c u r t}$ Interruption cost of load $l$ in time period $t$

$C_{t}^{i c} \quad$ Total inconvenience cost of all loads in the portfolio in time period $t$ 
$C_{l, t}^{\text {shift }}$ Shifting cost of shiftable load $l$ in time period $t$

\section{Functions}

$C_{l}^{\text {shift,tot }}$ Total shifting cost of shiftable load $l$

$V_{l, t}^{\text {base }} \quad$ Base marginal interruption cost of curtailable load $l$ in time period $t \quad[€ / \mathrm{kW}]$

$V_{l}^{\text {ref }} \quad$ Marginal interruption cost of load $l$ for a reference time of interruption $\quad[€ / \mathrm{kW}]$

$V_{l, t} \quad$ Marginal interruption cost of curtailable load $l$ in time period $t \quad[€ / \mathrm{kW}]$

\section{Parameters}

$E_{l}^{\text {shift }}$ Total energy consumption of shiftable load $l$

$f_{l, t}^{d} \quad$ Multiplication factor to take into account the type of day of interruption in time period $t$ for curtailable load $l$

$f_{l, t}^{s} \quad$ Multiplication factor to take into account the season of interruption in time period $t$ for curtailable load $l$

$f_{l, t}^{t} \quad$ Multiplication factor to take into account the time of interruption in time period $t$ for curtailable $\operatorname{load} l$

$P_{t}^{r e q}$ Load reduction required by the aggregator in time period $t$

$P_{l, t}^{B, c u r t}$ Baseline demand of curtailable load $l$ in time period $t$

$t_{l}^{a c t} \quad$ Earliest activation time period of shiftable load $l$

$\alpha_{l, t}^{\text {curt }}$ Quadratic parameter of marginal interruption cost function for curtailable load $l$ in time period $t$

$\alpha_{l}^{\text {shift }}$ Quadratic parameter of shifting cost function for shiftable load $l$

$\beta_{l, t}^{\text {curt }}$ Linear parameter of marginal interruption cost function for curtailable load $l$ in time period $t$

$\beta_{l}^{\text {shift }}$ Linear parameter of shifting cost function for shiftable load $l$

$\gamma_{l, t}^{\text {curt }}$ Constant parameter of marginal interruption cost function for curtailable load $l$ in time period $t$

$\gamma_{l}^{\text {shift }}$ Constant parameter of shifting cost function for shiftable load $l$

$\delta_{l}^{\text {shift,max }}$ Maximum delay of shiftable load $l$

$\Delta t \quad$ Length of time period 


\section{Introduction}

\subsection{Background}

Flexibility in end-consumers' electricity demand, including their ability to reduce their electricity consumption or defer the activation of some loads, can contribute to the secure and cost efficient operation of the power system. Studies of blackouts in 1996 and 2003 in the North-West American power system have for instance shown that shedding a relatively small amount of load could have avoided large-scale, uncontrolled electricity supply interruptions [1, 2]. In present, power systems' adequacy and security are increasingly challenged due to the massive integration of inverter-connected renewable energy sources [3]. Therefore, it is important to investigate how power system operation clothing dryers are examples of shiftable loads. Their operating cycle lasts over a predefined period and consumers can be flexible by delaying the activation of the cycle.

Although the involvement of readily-available, end-consumers' flexibility can contribute to the secure and cost-efficient operation of the power system, ensuring socially acceptable utilization of this experienced by the end-consumers. The inconvenience experienced by end-consumers due to load curtailment or delayed activation of appliances is dynamic and can be monitored using the notion of the inconvenience cost. The part of inconvenience caused by load curtailment has already been monitored using the interruption cost. Surveys have shown that the interruption cost depends on two [6]:

- Consumer characteristics representing the differentiated preferences of different consumers;

- Interruption characteristics, such as the time and the duration of interruptions. 
Besides the interruption cost, the inconvenience cost should contain the cost experienced due to the delayed activation of shiftable loads. A detailed model of the inconvenience cost enables system stakeholders to accurately assess the value of using distributed demand-side flexibility in system planning and operation.

\subsection{Motivation and contributions}

Although the inconvenience cost is an important factor impacting the efficiency and fairness of power system operation and demand response schemes, it has not yet been modelled in full detail by the existing literature. Table 1 gives an overview of the level of modelling detail of the inconvenience cost in existing studies. An important limitation of the existing models lies in their static nature. Static models prevent the integration of dynamic aspects, such as how the inconvenience cost is influenced by delayed activation of shiftable loads or the interruption duration. This means, for example, that existing models do not capture the difference in inconvenience costs associated with one interruption of two hours or two interruptions of one hour, for which it is intuitively clear that they result in a different inconvenience cost. Modelling the impact of the duration of interruption and the activation delay on the inconvenience cost requires that the dependence on the previous system state is captured. Static models do not contain internal history of states. This dependence can be incorporated in a dynamic model formulation.

In this context, this paper develops a dynamic model of the inconvenience cost experienced by endconsumers due to load curtailment and shifting. The proposed model makes the following contributions:

- The proposed model simultaneously takes into account five factors impacting the inconvenience cost: differentiated preferences of different consumer groups, the time of the interruption, the duration of the interruption, the differentiated valuation of different units of power and the temporal redistribution of shiftable loads. To the best of the authors' knowledge, none of the existing papers considers all these factors simultaneously.

- The proposed model is dynamic and future-agnostic, implying that it captures the time-coupling characteristics of consumers' flexibility and the temporal evolution of interruptions, without resorting to the unrealistic assumption that the time and the duration of interruptions are known in advance by the consumers. This is a significant improvement with respect to the static models used in the existing literature.

- The proposed model is quantitatively informed by data from publicly available consumers' surveys. These surveys specify the dependence of the inconvenience cost on the considered consumer group, the time of interruption and the duration of the interruption. Regarding aspects that have not yet been explored by surveys, including differentiated valuation of different units of power 


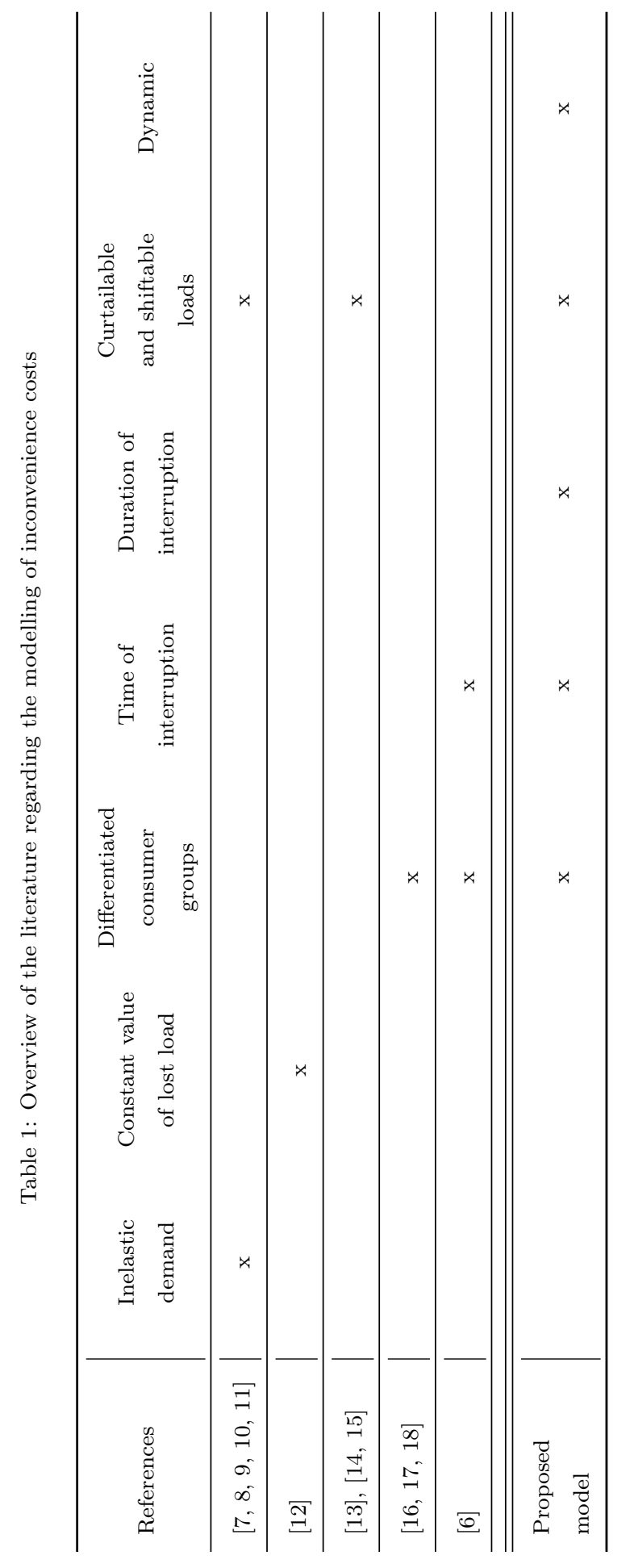


and the flexibility of shiftable loads, relevant assumptions are made and sensitivity analyses are carried out. The outcomes of simulations with the proposed model can specify the aspects that should be studied in more detail in future surveys to validate the assumptions and further exploit the potential of demand-side flexibility.

Case studies involve the application of the developed model by an aggregator who manages the flexible loads in its portfolio in a scenario where an adequacy issue arises in the system.

\subsection{Paper structure}

The rest of this paper is organized as follows. Section 2 introduces the generic formulation of the dynamic model of inconvenience experienced by end-consumers with different load types. Section 3 elaborates on the dynamic model of end-consumers' service reliability, whereas Section 4 focusses on the detailed modelling of the inconvenience cost. Section 5 demonstrates the use of the dynamic model in case studies involving portfolio management of an aggregator. Section 6 concludes the paper and gives directions for future work.

\section{Generic formulation of the dynamic model of inconvenience experienced by end- consumers due to load curtailment and shifting}

Load curtailment and delayed activation of shiftable appliances cause inconvenience to end-consumers. End-consumers' experienced inconvenience is influenced by dynamic aspects, such as the interruption duration and the time-coupling characteristics of shiftable loads, rendering it a continuous, dynamic system.

The dynamic system of end-consumers' experienced inconvenience can be modelled using a timevariant state-space input/output model, which can be generally expressed as:

$$
\begin{gathered}
\frac{d \mathbf{x}(t)}{d t}=g(\mathbf{x}(t), \mathbf{u}(t)) \\
y(t)=h_{t}(\mathbf{x}(t), \mathbf{u}(t))
\end{gathered}
$$

The vector of control variables $\mathbf{u}(t)$ of the dynamic system consists of the load curtailment and shifting actions at each time instant $t$, whereas the output $y(t)$ represents the inconvenience caused to the endconsumers at a certain time instant. The vector of physical state variables $\mathbf{x}(t)$ represents the reliability state of the consumer, which is determined by the interruption duration of curtailable loads and the activation delay of shiftable loads. Eq. (1) represents the dynamic model of the service reliability of an end-consumer and models how the service reliability level evolves over time. Eq. (2) models the inconvenience experienced by the end-consumers based on their service reliability level and the control 
actions taken. The function parameters of the output equation (Eq. (2)) are time dependent in order to capture the impact of the time of the interruption on the end-consumers' experienced inconvenience.

Although in theory inconvenience is a continuous function of time, the continuous nature of the system is approximated in the developed model by only looking at discrete time instants with fixed time intervals in between. Therefore, we assume that exogenous information is revealed and control actions $\mathbf{u}(t)$ are taken at discrete time instants. The discrete time instant control actions and exogenous information are linked to a specific time period according to the so-called information format to model time introduced in [19]. This format specifies that the discrete time index $t$ refers to the continuous time period from time instant $t-1$ up to and including time instant $t$. The experienced inconvenience at $t$ thus represents the inconvenience experienced during the respective time period. This results in a discrete time-variant state-space input/output model. The complete model is generically expressed as:

$$
\begin{gathered}
\mathbf{x}_{t}=g\left(\mathbf{x}_{t-1}, \mathbf{u}_{t}\right) \\
y_{t}=h_{t}\left(\mathbf{x}_{t}, \mathbf{u}_{t}\right)
\end{gathered}
$$

\section{Dynamic model of the service reliability of an end-consumer with different load types}

Consumers' experienced service reliability level is quantified in this paper by the interruption duration and activation delay of the consumer's loads. The interruption duration differs per load type: Curtailable loads are considered interrupted as soon as part of their required instantaneous power is not supplied, while shiftable loads are considered interrupted if their operating cycle cannot be completed by the latest allowable termination time. Furthermore, the activation delay is only relevant to shiftable loads. Therefore, different models are required for each load type, as detailed in following subsections.

\subsection{Curtailable loads}

The service reliability of a curtailable load is modelled using the interruption duration $D_{l, t}^{\text {int }}$ and an auxiliary variable to indicate whether the load is interrupted or not, i.e., the connection status $Y_{l, t}$. These variables can be considered as the state variables of curtailable loads. The connection status for curtailable loads is determined by the amount of power curtailed for that load $P_{l, t}^{\text {curt }}$ in a certain time period $t$. If (part of) the load is curtailed, i.e., $P_{l, t}^{\text {curt }}>0$, the load is assumed to be interrupted, i.e., 
$Y_{l, t}$ equals zero, as expressed by 1

$$
\left\{\begin{array}{r}
Y_{l, t}=1 \\
P_{l, t}^{\text {curt }}=0
\end{array}\right\} \underline{\vee}\left\{\begin{array}{r}
Y_{l, t}=0 \\
P_{l, t}^{\text {curt }}>0
\end{array}\right\} \quad \forall l \in \mathcal{L}^{\text {curt }}, t
$$

Based on the connection status, an auxiliary duration variable $D_{l, t}$ can be quantified, which represents the interruption duration $D_{l, t}^{i n t}$ if it is positive and the time since the last interruption $D_{l, t}^{n o r m}$ if it is negative. The variable changes sign if the connection status changes between two time periods, which is the case in the first two statements of the disjunction in Eq. (6) (The variable $D_{l, t-1}$ has the opposite sign than the variable $D_{l, t}$.). If the connection status does not change between two time periods, the variable $D_{l, t}$ is incremented. This is expressed in the last two statements of the disjunction in Eq. (6).

$$
\left\{\begin{array}{r}
D_{l, t}=\Delta t \\
D_{l, t-1}<0 \\
Y_{l, t}=0 \\
D_{l, t}^{\text {int }}=\Delta t \\
D_{l, t}^{\text {norm }}=0
\end{array}\right\} \underline{\vee}\left\{\begin{array}{r}
D_{l, t}=-\Delta t \\
D_{l, t-1}>0 \\
Y_{l, t}=1 \\
D_{l, t}^{i n t}=0 \\
D_{l, t}^{n o r m}=\Delta t
\end{array}\right\} \underline{v}\left\{\begin{array}{r}
D_{l, t}=D_{l, t-1}+\Delta t \\
D_{l, t-1}>0 \\
Y_{l, t}=0 \\
D_{l, t}^{i n t}=D_{l, t-1}+\Delta t \\
D_{l, t}^{\text {norm }}=0
\end{array}\right\} \underline{v}\left\{\begin{array}{r}
D_{l, t}=D_{l, t-1}-\Delta t \\
D_{l, t-1}<0 \\
Y_{l, t}=1 \\
D_{l, t}^{\text {int }}=0 \\
D_{l, t}^{\text {norm }}=-D_{l, t-1}+\Delta t
\end{array}\right\} \forall l, t
$$

In the first time period of the simulation, variables of the previous time period are not available and the dynamic model should be initialized. The initialization is based on the connection status in the first time period, expressed by Eq. (7).

$$
\left\{\begin{array}{r}
D_{l, t}=\Delta t \\
Y_{l, t}=0 \\
D_{l, t}^{\text {int }}=\Delta t \\
D_{l, t}^{\text {norm }}=0
\end{array}\right\} \underline{\vee}\left\{\begin{array}{r}
D_{l, t}=-\Delta t \\
Y_{l, t}=1 \\
D_{l, t}^{\text {int }}=0 \\
D_{l, t}^{\text {norm }}=\Delta t
\end{array}\right\} \quad \forall l, t=1
$$

\subsection{Shiftable loads}

The service reliability of shiftable loads is modelled using an activation variable and the delay of activation. The activation of a shiftable load is indicated by the binary activation variable $z_{l, t}$, which equals one at the time instant that the load is activated. Based on the activation variable, the activation status $Z_{l, t}$ is determined, equalling one once the load is activated (Eq. 12 and Eq. 13). The activation status of the shiftable load can be linked to the connection status $Y_{l, t}$. The link between

\footnotetext{
${ }^{1}$ The logical disjunction $A \underline{\vee} B$ is true, if $A$ is true or $B$ is true.
} 
the activation status and connection status depends on the time period. The activation of the shiftable load can be delayed within the time frame $T_{l}^{\text {shift }}=\left\{t_{l}^{a c t}, t_{l}^{a c t}+\delta_{l}^{\text {shift,max }}\right\}$. Before this time interval, the load is assumed to be connected (Eq. (9)), but not yet activated (Eq. (10)). As long as the load is not activated after the first possible activation time $t_{l}^{a c t}$ the auxiliary variable $Z_{l, t}$ equals zero and the load is assumed to be disconnected (Eq. (11)). If the load is activated, the activation variable $z_{l, t}$ equals one at the moment of activation. The activation and connection statuses equal one in the remaining time periods. If the cycle is shifted to be completed beyond the acceptable time frame, i.e., the activation time is after $t_{l}^{a c t}+\delta_{l}^{\text {shift,max }}$, the load is assumed to be curtailed. The indicator variable for curtailment $Q_{l, t}^{\text {shift }}$ equals one in this case (Eq. 15), while it equals zero until the latest allowable activation time (Eq. (14)).

$$
\begin{array}{ll}
Z_{l, t}=\{0,1\} & \forall l \in \mathcal{L}^{\text {shift }}, t \\
Y_{l, t}=1 & \forall l \in \mathcal{L}^{\text {shift }}, t<t_{l}^{a c t} \\
Z_{l, t}=0 & \forall l \in \mathcal{L}^{\text {shift }}, t<t_{l}^{a c t} \\
Y_{l, t}=Z_{l, t} & \forall l \in \mathcal{L}^{\text {shift }}, t \geq t_{l}^{\text {act }} \\
Z_{l, 1}=z_{l, t} & \forall l \in \mathcal{L}^{\text {shift }}, t=1 \\
Z_{l, t}=z_{l, t}+Z_{l, t-1} & \forall l \in \mathcal{L}^{\text {shift }}, t>1 \\
Q_{l, t}^{\text {shift }}=0 & \forall l \in \mathcal{L}^{\text {shift }}, t \leq t_{l}^{\text {act }}+\delta_{l}^{\text {shift }, \text { max }} \\
Q_{l, t}^{\text {shift }}=1-Z_{l, t} & \forall l \in \mathcal{L}^{\text {shift }}, t>t_{l}^{\text {act }}+\delta_{l}^{\text {shift,max }}
\end{array}
$$

The constraints for the interruption duration specified in Eq. (6) and Eq. (7) are used to define the activation delay and the interruption duration of shiftable loads. Within the acceptable time frame $T_{l}^{\text {shift }}$, load activation can be delayed. The activation delay $\delta_{l, t}^{\text {shift }}$ is calculated based on the interruption duration $D_{l, t}^{i n t}$ specified in Eq. (6) using the connection status of the shiftable load and the interruption duration $D_{l, t}^{\text {int }, \text { shift }}$ equals zero:

$$
\begin{array}{ll}
\delta_{l, t}^{\text {shift }}=D_{l, t}^{\text {int }} & \forall l \in \mathcal{L}^{\text {shift }}, t \leq t_{l}^{\text {act }}+\delta_{l}^{\text {shift,max }} \\
D_{l, t}^{\text {int } t \text { shift }}=0 & \forall l \in \mathcal{L}^{\text {shift }}, t \leq t_{l}^{\text {act }}+\delta_{l}^{\text {shift }, \text { max }}
\end{array}
$$

If the load is activated beyond the acceptable time frame, the variable $D_{l, t}^{i n t}$ keeps on incrementing until the load is activated. The interruption duration of the shiftable load $D_{l, t}^{\text {int,shift }}$ equals the delay of activation beyond the acceptable time interval. This value is obtained if the length of the time interval of acceptable activation, i.e., $\delta_{l}^{\text {shift,max }}+\Delta t$, is subtracted from the total delay counted in $D_{l, t}^{i n t}$, as specified in Eq. 18 .

$$
D_{l, t}^{\text {int,shift }}=\max \left[0, D_{l, t}^{\text {int }}-\left(\delta_{l}^{\text {shift }, \text { max }}+\Delta t\right)\right] \quad \forall l \in \mathcal{L}^{\text {shift }}, t>t_{l}^{a c t}+\delta_{l}^{\text {shift }, \text { max }}
$$




\section{Modelling end-consumers' experienced inconvenience cost}

The key output variable of the model introduced in Eq. (3) and (4) is the inconvenience cost experienced in a portfolio of loads in time period $t$. The inconvenience cost consists of the cost attributed to the inconvenience experienced due to delayed activation of shiftable loads and the cost attributed to the interruption of curtailable loads or overtime activation of shiftable loads. To a certain

Based on the Norwegian survey results, a piece-wise linear function seems to appropriately model the relation between the interruption duration and the total interruption cost per unit of interrupted power per consumer group [22, 23, 24]. This model is developed for a reference time instant. The total interruption cost is normalized on the total power curtailed, implying the assumption that each unit of power in the consumer's portfolio is equally valued. The derivative of the total normalized interruption cost with respect to the interruption duration expresses the marginal interruption $\operatorname{cost} V_{l}\left(D^{i n t}\right)$ as a continuous function of the interruption duration. This represents the average cost of an additional hour of interruption of a unit of power at the reference time instant. The average interruption cost

\footnotetext{
${ }^{2}$ Ovaere et al. give an overview of the level of detail of VOLL data collected in different countries 6 .

${ }^{3}$ The average marginal interruption cost is an approximation of the real marginal interruption cost of each unit of power, as it assumes each individual unit of power to be equally valued.
} 
of a $1 \mathrm{~kW}$ interruption attributable to an additional time period in our discretized model equals the integral of the marginal interruption cost over the given time period, as given in Eq. 19.

$$
V_{l}^{r e f}\left(D_{l, t}^{i n t}\right)=\int_{D_{l, t}^{i n t}-\Delta t}^{D_{l, t}^{i n t}} V_{l}\left(D^{i n t}\right) \cdot d D^{i n t}=\frac{V_{l}\left(D_{l, t}^{i n t}\right)+V_{l}\left(D_{l, t}^{i n t}-\Delta t\right)}{2} \cdot \Delta t \quad \forall l, t
$$

As the aforementioned function determines the average marginal interruption cost for a reference time instant, the determined reference marginal interruption cost should be modified to account for the effective time of the interruption. The Norwegian surveys have derived multiplication factors to capture the impact of the time of interruption [22]. The multiplication factors make a distinction between four consumers groups, the season $\left(f_{l, t}^{s}\right)$, type of day $\left(f_{l, t}^{d}\right)$ and time of day $\left(f_{l, t}^{t}\right)$, as presented in Table 2, Combining the reference marginal interruption cost calculated using Eq. 19p with the

Table 2: Multiplication factors to consider the impact of the time of interruption for different consumer groups in the interruption cost [22, Table A and Table B]. The multiplication factors for agriculture are estimated based on [24].

\begin{tabular}{ll|ccccc}
\hline & & Residential & Industry & Commercial & Public & Agriculture \\
\hline \multirow{3}{*}{ Season $f_{l, t}^{s}$} & Winter & 1 & 1 & 1 & 1 & 1 \\
& Spring & 0.57 & 0.87 & 1 & 0.67 & 0.67 \\
& Summer & 0.44 & 0.86 & 1.02 & 0.51 & 0.51 \\
& Autumn & 0.75 & 0.88 & 1.06 & 0.58 & 0.58 \\
\hline \multirow{2}{*}{ Day $f_{l, t}^{d}$} & Weekday & 1 & 1 & 1 & 1 & 1 \\
& Saturday & 1.07 & 0.13 & 0.45 & 0.3 & 0.3 \\
& Sunday & 1.07 & 0.14 & 0.11 & 0.29 & 0.29 \\
\hline \multirow{3}{*}{ Time $f_{l, t}^{t}$} & Night & 0.4 & 0.12 & 0.11 & 0.43 & 0.43 \\
& Morning & 0.69 & 1 & 1 & 1 & 1 \\
& Evening & 1 & 0.14 & 0.29 & 0.31 & 1 \\
\hline
\end{tabular}

multiplication factors in Table 2, we determine for a particular time and duration of interruption the average marginal cost of interrupting a unit of power $V_{l, t}^{\text {base }}\left(D_{l, t}^{\text {int }}\right)$ in our discretized model, as expressed by:

$$
V_{l, t}^{\text {base }}\left(D_{l, t}^{\text {int }}\right)=V_{l}^{\text {ref }}\left(D_{l, t}^{\text {int }}\right) \cdot f_{l, t}^{s} \cdot f_{l, t}^{d} \cdot f_{l, t}^{t} \quad \forall l \in \mathcal{L}^{\text {curt }}, t
$$

\subsection{Valuation of different units of power}

Applying the average marginal interruption cost function derived in Eq. 20 implies the assumption that each unit of power is equally valued, irrespectively of the specific appliance it is consumed by and the specific service it provides to the consumers. Nevertheless, consumers have more and less critical 
appliances in their portfolio: a medical appliance is for instance more critical than a television. In order to capture this differentiated valuation of different appliances, the interruption cost of different units of power should be varied. Therefore, the marginal interruption cost is modelled as a function of the amount of power curtailment.

Karimi has proposed to model the marginal benefit of supplying load as a second-order polynomial function [25. We have applied a similar reasoning to use a second-order polynomial function to model the marginal interruption cost $V_{l, t}\left(P^{c u r t}\right)$ (Eq. (21)). This polynomial function should satisfy some boundary conditions to comply with rationality constraints. First of all, $V_{l, t}\left(P^{c u r t}\right)$ should be nonnegative, implying that an increasing interruption cost is experienced if more load is curtailed (Eq. (22)). Secondly, the derivative of $\frac{d V_{l, t}\left(P^{\text {curt }}\right)}{d P^{\text {curt }}}$ should also be non-negative to capture the fact that additional curtailment affects load that is equally or higher valued (Eq. (23)). Thirdly, the mean of the marginal interruption cost function equals the average marginal interruption cost $V_{l, t}^{\text {base }}\left(D_{l, t}^{\text {int }}\right)$, as determined in the previous subsection (Eq. 24) ).

Given these constraints, this function can capture different consumers' perceptions and characteristics. Three different sets of consumers are explored in this paper as an example: consumers with high flexibility (perceiving most of their appliances as non-critical appliances), consumers with low flexibility (perceiving most of their appliances as critical) and consumers with medium flexibility (intermediate case). High, medium and low flexibility are modelled through a convex, linear and concave function, respectively Eq. (25), 26, and (27).

$$
\begin{aligned}
& V_{l, t}\left(P^{\text {curt }}\right)=\alpha_{l, t}^{\text {curt }} \cdot\left(P^{\text {curt }}\right)^{2}+\beta_{l, t}^{\text {curt }} \cdot P^{\text {curt }}+\gamma_{l, t}^{\text {curt }} \quad \forall l \in \mathcal{L}^{\text {curt }}, t \\
\text { With: } & V_{l, t}\left(P^{\text {curt }}\right) \geq 0 \\
& \frac{d V_{l, t}\left(P^{\text {curt }}\right)}{d P^{\text {curt }}} \geq 0 \\
& \frac{1}{P_{l, t}^{B, \text { curt }}} \int_{0}^{P_{l, t}^{B, \text { curt }}} V_{l, t}\left(P^{\text {curt }}\right) d P^{\text {curt }}=V_{l, t}^{\text {base }}\left(D_{l, t}^{\text {int }}\right) \\
& \text { High flexibility: } \frac{d^{2} V_{l, t}\left(P^{\text {curt }}\right)}{d\left(P^{c u r t}\right)^{2}} \geq 0 \\
& \text { Medium flexibility: } \frac{d^{2} V_{l, t}\left(P^{\text {curt }}\right)}{d\left(P^{c u r t}\right)^{2}}=0 \\
& \text { Low flexibility: } \frac{d^{2} V_{l, t}\left(P^{c u r t}\right)}{d\left(P^{c u r t}\right)^{2}} \leq 0
\end{aligned}
$$

Based on the above discussion, the interruption cost function parameters $\alpha_{l, t}^{\text {curt }}, \beta_{l, t}^{\text {curt }}$ and $\gamma_{l, t}^{\text {curt }}$ for consumers with different levels of flexibility are summarized in Table $3 . V_{l, t}^{\text {base }}\left(D_{l, t}^{\text {int }}\right)$ in Table 3 is modelled as in Eq. 20.

$V_{l, t}\left(P^{\text {curt }}\right)$ expresses the interruption cost attributed to an additional unit of curtailed power for a particular time period $t$ and duration of interruption $D_{l, t}^{i n t}$. The total interruption cost associated with 
Table 3: Interruption cost function parameters for consumers with different levels of flexibility

\begin{tabular}{c|ccc}
\hline & High & Medium & Low \\
\hline$\alpha_{l, t}^{\text {curt }}$ & $3 \cdot \frac{V_{l, t}^{\text {base }}\left(D_{l, t}^{\text {int }}\right)}{\left(P_{l, t}^{B, c u r t}\right)^{2}}$ & 0 & $-\frac{3 \cdot V_{l, t}^{\text {base }}\left(D_{l, t}^{\text {int }}\right)}{2 \cdot\left(P_{l, \text { curt }}^{B, c u}\right)^{2}}$ \\
$\beta_{l, t}^{\text {curt }}$ & 0 & $2 \cdot \frac{V_{l, t}^{\text {base }}\left(D_{l, t}^{\text {int }}\right)}{\left(P_{l, t}^{B, c u r t}\right)}$ & $3 \cdot \frac{V_{l, t}^{\text {base }}\left(D_{l, t}^{\text {int }}\right)}{\left(P_{l, t}^{B, \text { curt }}\right)}$ \\
$\gamma_{l, t}^{\text {curt }}$ & 0 & 0 & 0 \\
\hline
\end{tabular}

a time period $t$ due to the curtailment of $P_{l, t}^{\text {curt }}$ for a particular interruption duration $D_{l, t}^{\text {int }}$ equals the integral:

$$
\begin{aligned}
C_{l, t}^{\text {curt }} & =\int_{0}^{P_{l, t}^{\text {curt }}} V_{l, t}\left(P^{\text {curt }}\right) \cdot d P^{\text {curt }} & \\
& =\alpha_{l, t}^{\text {curt }} \cdot \frac{\left(P_{l, t}^{\text {curt }}\right)^{3}}{3}+\beta_{l, t}^{\text {curt }} \cdot \frac{\left(P_{l, t}^{\text {curt }}\right)^{2}}{2}+\gamma_{l, t}^{\text {curt }} \cdot P_{l, t}^{\text {curt }} & \forall l, t
\end{aligned}
$$

\subsection{Shifting cost}

The shifting cost monetizes the inconvenience experienced by end-consumers due to the delayed activation of shiftable loads within the predefined, acceptable time interval $\left\{t_{l}^{a c t}, t_{l}^{a c t}+\delta_{l}^{s h i f t, \max }\right\}$. Delayed activation deteriorates the level of service provided to the consumers (e.g., clean clothes being available later), which comes at a cost. Karimi has modelled the relation between the activation delay and the shifting cost as a second-order polynomial function (Eq. (29) [25]. This function should satisfy some boundary conditions to comply with rationality constraints. First of all, the shifting cost equals zero if the load is activated at $t_{l}^{a c t}$, which corresponds to a case of no delay in activation (Eq. (30). Secondly, if the maximal delay is reached and the load is not activated within the acceptable time interval, the total shifting cost is equal to the interruption cost associated with the total energy requirement of the cycle of the load (Eq. (31)). The interruption cost is determined based on the average marginal interruption cost of Eq. 19p, because each additional unit of power shifted is equally valued. This is the case because shiftable loads have a fixed power profile that should be considered as a single appliance of which the activation is delayed. Thirdly, as the shifting cost increases with the delay, the first derivative of the shifting cost should be non-negative (Eq. (32)).

Given these constraints, this function can capture different consumers' perceptions and characteristics. Three different sets of consumers are explored in this paper as an example: consumers with early activation preferences (who do not want for instance to be bothered with emptying the washing machine late in the evening), consumers with late activation preferences (who come for instance home late and they do not want their clothes to be wet in the washing machine for too long) and consumers with indifferent activation preference (intermediate case). Early, late and indifferent activation preferences are modelled through a concave, convex and linear function of the shifting cost, respectively Eq. 
(33), Eq. 34) and Eq. (35).

$$
C_{l}^{\text {shift,tot }}\left(\delta^{\text {shift }}\right)=\alpha_{l}^{\text {shift }} \cdot\left(\delta^{\text {shift }}\right)^{2}+\beta_{l}^{\text {shift }} \cdot \delta^{\text {shift }}+\gamma_{l}^{\text {shift }} \quad \forall l \in \mathcal{L}^{\text {shift }}, t
$$

with: $C_{l}^{\text {shift,tot }}(0)=0$

$$
\begin{aligned}
& C_{l}^{\text {shift,tot }}\left(\delta_{l}^{\text {shift, max }}\right)=V_{l}^{\text {ref }}(\Delta t) \cdot E_{l}^{\text {shift }} \\
& \frac{\partial C_{l}^{\text {shift }, \text { tot }}\left(\delta^{\text {shift }}\right)}{\partial \delta^{\text {shift }}} \geq 0
\end{aligned}
$$

Early activation: $\frac{\partial^{2} C_{l}^{\text {shift,tot }}\left(\delta^{\text {shift }}\right)}{\partial\left(\delta^{\text {shift }}\right)^{2}} \leq 0$

Late activation: $\frac{\partial^{2} C_{l}^{\text {shift,tot }}\left(\delta^{\text {shift }}\right)}{\partial\left(\delta^{\text {shift }}\right)^{2}} \geq 0$

Indifferent: $\frac{\partial^{2} C_{l}^{\text {shift,tot }}\left(\delta^{\text {shift }}\right)}{\partial\left(\delta^{\text {shift }}\right)^{2}}=0$

Based on the above discussion, the shifting cost function parameters $\alpha_{l}^{\text {shift }}, \beta_{l}^{\text {shift }}$ and $\gamma_{l}^{\text {shift }}$ for consumers with different activation preferences are summarized in Table 4.

Table 4: Shifting cost function parameters for consumers with different activation preferences

\begin{tabular}{l|ccc}
\hline Activation preferences & Early & Indifferent & Late \\
\hline$\alpha_{l}^{\text {shift }}$ & $\frac{V_{l}^{\text {ref }}(\Delta t) \cdot E_{l}^{\text {shift }}}{\left(\delta_{l}^{\text {shift }, \text { max }}\right)^{2}}$ & 0 & $-\frac{V_{l}^{\text {ref }}(\Delta t) \cdot E_{l}^{\text {shift }}}{\left(\delta_{l}^{\text {shift }, \text { max }}\right)^{2}}$ \\
$\beta_{l}^{\text {shift }}$ & 0 & $\frac{V_{l}^{\text {ref }}(\Delta t) \cdot E_{l}^{\text {shift }}}{\delta_{l}^{\text {shift }, \text { max }}}$ & $2 \cdot \frac{V_{l, t}^{\text {ref }}(\Delta t) \cdot E_{l}^{\text {shift }}}{\delta_{l}^{\text {shift }, \text { max }}}$ \\
$\gamma_{l}^{\text {shift }}$ & 0 & 0 & 0 \\
\hline
\end{tabular}

Whereas the steady-state model with full knowledge of the future in 25] counts the total shifting cost at the activation time, a future-agnostic, dynamic model of inconvenience should attribute to each time period $t$ the shifting cost experienced due to the additional delay in time period $t$. The cost of an additional unit of delay for a given delay equals the derivative of the total shifting cost:

$$
C_{l}^{\text {shift,tot }}\left(\delta^{\text {shift }}\right)=\frac{\partial C_{l}^{\text {shift }, \text { tot }}\left(\delta^{\text {shift }}\right)}{\partial \delta^{\text {shift }}}=2 \cdot \alpha_{l}^{\text {shift }} \cdot \delta^{\text {shift }}+\beta_{l}^{\text {shift }} \quad \forall l \in \mathcal{L}^{\text {shift }}, t
$$

Given the employed discretization of time, the shifting cost incurred at period $t$ equals the integral of $C_{l}^{\text {shift,tot }}\left(\delta^{\text {shift }}\right)$ over the delay experienced in this period:

$C_{l, t}^{\text {shift }}=\int_{\delta_{l, t}^{\text {shift }}-\Delta t}^{\delta_{l, t}^{\text {shift }}} C_{l}^{\text {shift }, \text { tot }}\left(\delta^{\text {shift }}\right) \cdot d \delta^{\text {shift }}=\frac{C_{l}^{\text {shift }, \text { tot }}\left(\delta_{l, t}^{\text {shift }}\right)+C_{l}^{\text {shift }, \text { tot }}\left(\delta_{l, t}^{\text {shift }}-\Delta t\right)}{2} \cdot \Delta t \quad \forall l \in \mathcal{L}^{\text {shift }}, t$

\subsection{Total inconvenience cost}

The total inconvenience cost experienced by a portfolio of loads in a certain time period consists of different cost components representing the inconvenience associated with different types of loads in this 
portfolio. First of all, the curtailment of curtailable loads in a certain time period results in an interruption cost in this time period. The experienced interruption cost for this period $C_{l, t}^{\text {curt }}\left(P_{l, t}^{\text {curt }}, D_{l, t}^{\text {int }}\right)$ can be calculated as in Eq. (28). Secondly, the inconvenience cost associated with the delayed activation of shiftable loads equals the shifting $\operatorname{cost} C_{l, t}^{\text {shift }}\left(\delta_{l, t}^{\text {shift }}\right)$ within the acceptable time interval $T_{l}^{\text {shift }}$ and the interruption cost $C_{l, t}^{\text {curt }}\left(E_{l}^{\text {shift }}, D_{l, t}^{\text {int,shift }}\right)$ beyond the acceptable time interval. $C_{l, t}^{\text {shift }}\left(\delta_{l, t}^{\text {shift }}\right)$ is calculated according to Eq. (37), whereas $C_{l, t}^{\text {curt }}\left(E_{l}^{\text {shift }}, D_{l, t}^{\text {int,shift }}\right)$ is calculated according to Eq. 28) and the marginal interruption cost $V_{l}^{r e f}$. The resulting total inconvenience $\operatorname{cost} C_{t}^{i c}$ for time period $t$ equals:

$$
\begin{aligned}
C_{t}^{i c}= & \sum_{l \in \mathcal{L}^{\text {curt }}} C_{l, t}^{\text {curt }}\left(P_{l, t}^{\text {curt }}, D_{l, t}^{\text {int }}\right)+ \\
& \sum_{l \in \mathcal{L}^{\text {shift }}}\left[\left(1-Z_{l, t}\right) \cdot C_{l, t}^{\text {shift }}\left(\delta_{l, t}^{\text {shift }}\right) \cdot \mathbb{1}_{T_{l}^{\text {shift }}}(t)+Q_{l, t}^{\text {shift }} \cdot C_{l, t}^{\text {curt }}\left(E_{l}^{\text {shift }}, D_{l, t}^{\text {int }, \text { shift }}\right)\right] \quad \forall t
\end{aligned}
$$

Where $\mathbb{1}_{T_{l}^{\text {shift }}}(t)$ is the indicator function that equals one if $t$ is part of the acceptable time interval $T_{l}^{\text {shift }}$ and zero otherwise.

\subsection{Model implementation}

In order to formulate the proposed model as an optimization problem, the logical disjunctions have been transformed to closed-form expressions, using disjunctive programming. Specifically, the logical disjunctions represent an exclusive OR condition, i.e., $A \vee B$ is true, if and only if $A$ is true or $B$ is true with $A$ and $B$ generic sets of conditions. This OR condition is transformed to a closed-form expression through the employment of binary variables $d_{A}$ and $d_{B}$, indicating whether the sets $\mathrm{A}$ and $\mathrm{B}$, respectively, are true, i.e., the corresponding binary variable equals 1 , or not, i.e., the binary variable equals 0 (Eq. 39 and (40). Eq. (41) ensures that both sets of conditions cannot be true simultaneously.

$$
\begin{aligned}
& \text { A } B \\
& \left\{\begin{array}{l}
A_{1} \\
A_{2}
\end{array}\right\} \underline{\vee}\left\{\begin{array}{c}
B_{1} \\
B_{2}
\end{array}\right\} \\
& d_{A}, d_{B} \in\{0,1\} \\
& d_{A}+d_{B}=1
\end{aligned}
$$

The conditions of each generic set A and B are related through a logical AND condition, e.g., A is true if $A_{1}$ is true and $A_{2}$ is true. This AND condition is transformed to a closed-form expression through the employment of binary variables $d_{A 1}$ and $d_{A 2}$ indicating whether the conditions $A_{1}$ and $A_{2}$, respectively, are true. This implies that the AND condition can be expressed through a min operator 
(Eq. $46-(47))^{4}$

$$
\begin{aligned}
& A_{1} \text { true } \rightarrow d_{A 1}=1 \\
& A_{2} \text { true } \rightarrow d_{A 2}=1 \\
& B_{1} \text { true } \rightarrow d_{B 1}=1 \\
& B_{2} \text { true } \rightarrow d_{B 2}=1 \\
& d_{A}=\min \left\{d_{A 1}, d_{A 2}\right\} \\
& d_{B}=\min \left\{d_{B 1}, d_{B 2}\right\} \\
& d_{A 1}, d_{A 2}, d_{B 1}, d_{B 2} \in\{0,1\}
\end{aligned}
$$

This min operator can be expressed in closed-form as (for the example of Eq. (46)):

$$
\begin{aligned}
& 0 \leq d_{A} \\
& d_{A 1}+d_{A 2}-1 \leq d_{A} \\
& d_{A} \leq d_{A 1} \\
& d_{A} \leq d_{A 2}
\end{aligned}
$$

\section{Case study: Portfolio management of an aggregator}

\subsection{Description}

Although the proposed model of consumers' inconvenience can be potentially used in numerous different applications, this paper applies it in the context of optimizing the management of a portfolio of flexible loads by an aggregator. Specifically, assuming that an adequacy issue emerges in the system, the aggregator is instructed by the system operator to reduce the total demand of its portfolio by a specific amount $P_{t}^{r e q}$ over a set of periods $t$. The aggregator applies the proposed model to distribute the requested load reduction among different consumers and loads in its portfolio, with the objective of minimizing the total inconvenience cost of its consumers.

The focus of the examined studies lies in analyzing the resulting distribution of the requested load reduction and the resulting total inconvenience cost, when varying levels of modelling detail are incorporated in the representation of the consumers' inconvenience. These varying levels of detail correspond to the different factors discussed in previous parts of the paper, including differentiated preferences of different consumer groups, the time of the interruption, the duration of the interruption, the differentiated valuation of different curtailable loads and the temporal redistribution of shiftable

\footnotetext{
${ }^{4}$ References [26, 27, 28, explain how to translate the disjunctions and other logical constraints to closed-form formulations. $A_{1}$ true $\rightarrow d_{A 1}=1$ has the meaning if $A_{1}$ is true, then $d_{A 1}=1$.
} 
loads. The distribution of load reductions resulting from the inconvenience cost minimization is applied to the end-consumers and determines their experienced inconvenience cost. End-consumers' experienced inconvenience for given control actions $P_{l, t}^{\text {curt }}$ and $z_{l, t}$ is determined using the detailed dynamic model of the inconvenience cost, incorporating all factors. By analyzing the results, the impacts of considering these complex factors in the aggregator's decision making through the proposed model are quantified.

\subsection{Data}

The examined studies are carried out on a test system resembling the Belgian system and its loadshedding plan for the winter of 2014-2015. The reference total electricity demand of the Belgian system is $13,120 \mathrm{MW}$ and is distributed over five geographical zones and seven slices, as illustrated in Fig. 1 . The assumed adequacy issue lasts for five hours between 16:00 and 21:00 on a winter weekday and the total demand in this interval is assumed equal to the above reference value. A resolution of one hour is selected for the executed simulations.

\begin{tabular}{ccccccc}
\hline \multicolumn{7}{c}{ Geographical zones } \\
Slices & NW & NE & CE & SW & SE & TOTAL \\
\hline \hline 1 & 130 & 130 & 130 & 65 & 65 & $520 \mathrm{MW}$ \\
\hline 2 & 130 & 130 & 130 & 65 & 65 & $520 \mathrm{MW}$ \\
\hline 3 & 130 & 130 & 130 & 65 & 65 & $520 \mathrm{MW}$ \\
\hline 4 & 130 & 130 & 130 & 65 & 65 & $520 \mathrm{MW}$ \\
\hline 5 & 130 & 130 & 130 & 65 & 65 & $520 \mathrm{MW}$ \\
\hline 6 & 130 & 130 & 130 & 65 & 65 & $520 \mathrm{MW}$ \\
\hline 7 & 2500 & 2500 & 2500 & 1250 & 1250 & $10000 \mathrm{MW}$ \\
\hline TOTAL & & & & & $13120 \mathrm{MW}$ \\
\hline
\end{tabular}

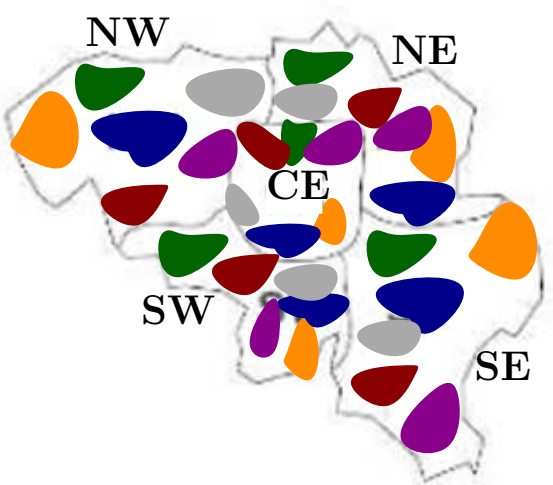

Figure 1: The division of Belgium into geographical zones and slices according to the load-shedding plan in the winter of 2014-2015 (Data: Elia). The division in slices is illustrative. Slice 7 corresponds to the white areas.

The portfolio of the examined aggregator includes $0.01 \%$ of the total demand in zone SE, and is distributed over the 7 slices according to the distribution of Fig. 1 The total load reduction requested from the aggregator is equal to $8.27,7.29,7.29,7.81$ and $7.81 \mathrm{~kW}$ over the five hours of the adequacy issue. The aggregator's portfolio consists of a discrete number of end-consumers that are categorized in five consumer groups, i.e., industry, residential, commercial, public and agriculture. The distribution of the end-consumers over the slices of the load-shedding plan and their respective load are summarized in Table 5. Residential consumers have curtailable and shiftable load, whereas the other consumer groups only have curtailable loads. Curtailable load is modelled through a continuous variable per consumer, whereas shiftable load is modelled as a single appliance per consumer, the cycle of which 
entails a fixed power profile and its activation can be delayed. The cycle of this shiftable appliance lasts one hour. In scenarios that omit the possibility of load shifting, the shiftable load is considered as part of the curtailable load and is operated in the first time period.

The calculation of the inconvenience cost is based on VOLL data collected by surveys in Norway. To the best of the authors' knowledge, this is the most detailed VOLL data available and its transparent data format can serve as an example for similar surveys in other countries. The marginal interruption cost for a reference time of interruption $V_{l}^{\text {ref }}\left(D_{l, t}^{\text {int }}\right)$ employed in the case studies is summarized in Table 6 ,

Six scenarios are considered in the case study. Scenarios 1-4 are defined based on available data: (i) a rule-based approach based on the Belgian load-shedding plan of 2014-2015, (ii) differentiation between consumer groups, (iii) impact of the time of interruption and (iv) impact of duration of interruption. Scenarios 5 and 6 assess the impact of the valuation of different units of power and the temporal redistribution of shiftable load in the aggregator's portfolio.
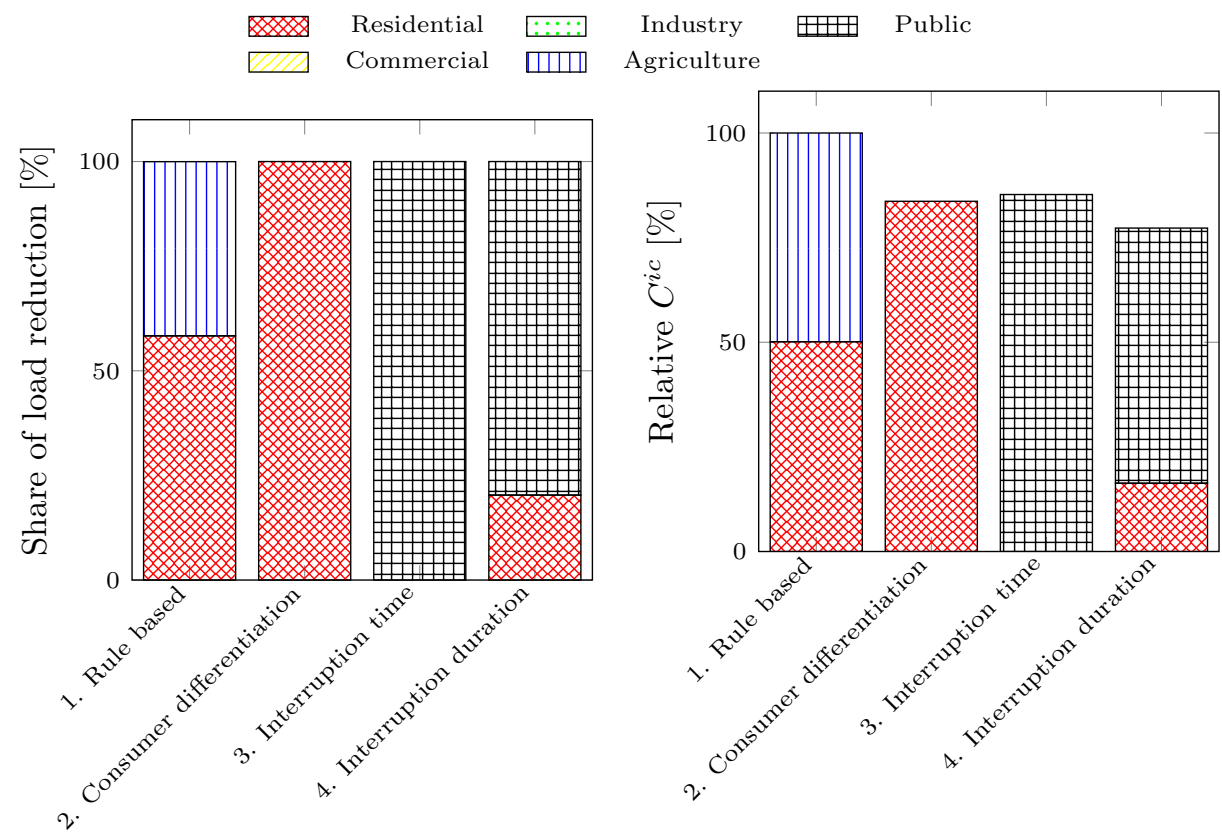

(a) Distribution of load reduction

(b) Relative total inconvenience cost (with respect to rule-based approach)

Figure 2: Distribution of load reduction (a) and total inconvenience cost (b) over the aggregator's portfolio in scenarios $1-4$.

\subsection{Scenario 1: Rule-based approach based on Belgian load-shedding plan}

The current paradigm for dealing with adequacy issues in the Belgian system involves a rulebased approach of rolling blackouts that does not explicitly consider (and thus does not minimize) the 


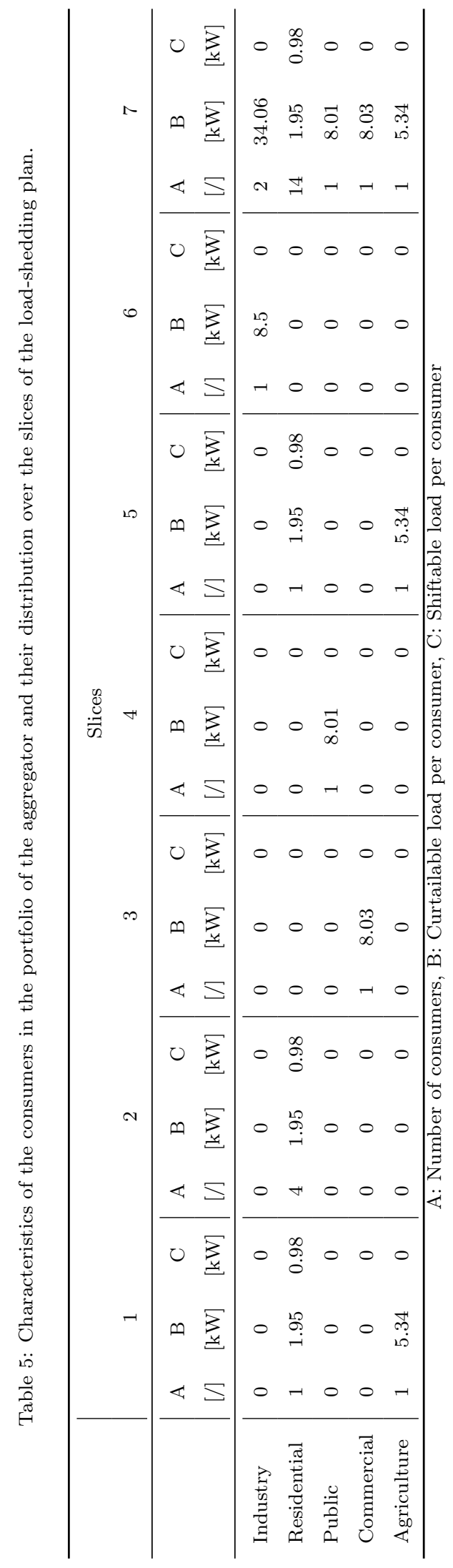


Table 6: Reference marginal interruption $\operatorname{cost} V_{l}^{r e f}\left(D_{l, t}^{i n t}\right)[€ / \mathrm{kW}]$ as a function of the interruption duration applied in the case study and based on the Norwegian VOLL data in 22 .

\begin{tabular}{c|ccccc}
\hline & Residential & Industry & Commercial & Public & Agriculture \\
\hline $0<D_{l, t}^{\text {int }}<4$ hours & 1.09 & 8.52 & 9.43 & 2.88 & 1.62 \\
$4 \leq D_{l, t}^{\text {int }}<8$ hours & 1.32 & 5.76 & 14.63 & 5.37 & 1.48 \\
\hline
\end{tabular}

consumers' inconvenience cost. Specifically, slices 1-6 of Fig. 11 are interrupted with an alternating sequence, while slice 7 is never interrupted (assuming that it represents highly critical load). Each interruption lasts for a maximum duration of three hours.

Since the examined adequacy issue lasts for 5 hours, this rule-based approach implies that slice 1 is interrupted in the first three hours and slice 2 is interrupted in the last two hours. Based on Table 5. this means that part of the demand of residential and agricultural consumers is reduced, as also illustrated in Fig. 2a. Since this approach does not consider the inconvenience cost and any factors affecting it, it yields the highest inconvenience cost, as illustrated in Fig. 2b where the inconvenience cost of this rule-based approach is used as the reference.

The following scenarios move away from the rule-based approach of scenario 1, and employ the proposed model to minimize the inconvenience cost of the aggregator's portfolio.

\subsection{Scenario 2: Differentiation between consumer groups}

Scenario 2 accounts for the differentiated preferences of different consumer groups. All the load of a particular consumer is equally valued according to a constant marginal interruption cost, but this marginal interruption cost differs per consumer group, according to the values presented in the first row of Table 7 that summarizes the average marginal cost of interrupting a unit of power per consumer group and per scenario for scenarios 2 - 4 of the case study. As the impact of the time and duration of the interruption is not considered in scenario 2, these values correspond to the reference marginal interruption cost for an interruption of one hour presented in Table 6 .

Given that the marginal interruption cost of the residential consumers is the lowest one in Scenario 2 (Table 7), the aggregator uses only these consumers to satisfy the requested load reduction, as illustrated in Fig. 2a. Since the marginal interruption cost of residential consumers is smaller than the one of agricultural consumers that also contribute to the requested load reduction in Scenario 1, Scenario 2 yields a lower inconvenience cost, as illustrated in Fig. $2 \mathrm{~b}$

\subsection{Scenario 3: Consideration of the time of interruption}

On top of the differentiated preferences of different consumer groups, scenario 3 accounts for the impact of the time of interruption. Therefore, the marginal interruption cost of the different consumer 
Table 7: Average marginal cost of interrupting a unit of power $[€ / \mathrm{kW}]$ per consumer group and per scenario for scenarios $2-4$ in the case study

\begin{tabular}{l|ccccc}
\hline & Residential & Industry & Commercial & Public & Agriculture \\
\hline Scenario 2 & $\mathbf{1 . 0 9}$ & 8.52 & 9.43 & 2.88 & 1.62 \\
Scenario 3 & 1.09 & 1.19 & 2.74 & $\mathbf{0 . 8 9}$ & 1.62 \\
\hline Scenario 4 & & & & & \\
$0 \leq D^{\text {int }}<4$ hours & 1.09 & 1.19 & 2.74 & 0.89 & 1.62 \\
$4 \leq D^{\text {int }}<8$ hours & 1.32 & 0.81 & 4.24 & 1.66 & 1.48 \\
\hline
\end{tabular}

groups considered in scenario 2 is multiplied by the multiplication factors applying to the time of the assumed interruption (winter, weekday, evening), based on Table 2 and Eq. 200. The resulting row of Table 7

The consideration of the duration of interruption changes the situation with respect to scenarios 2 and 3 where the whole burden of load reduction is laid on a single consumer group, as illustrated in Fig. 2a. Specifically, the aggregator uses the public consumers during the first hours of the interruption, but for extended interruptions of four hours or more (Table 7). This change of affected consumer groups 
over time results from the combined modelling of the differentiation between consumer groups, time of interruption and duration of interruption, and yields the lowest inconvenience cost among the four scenarios considered so far, as illustrated in Fig. 2b. These outcomes are driven by the dynamic nature of the proposed model and cannot be captured by state-of-the-art, static models.

\subsection{Scenario 5: Valuation of different units of power}

While scenarios 1 - 4 assume that all units of power are equally valued by each consumer, scenario 5 accounts for the differentiated valuation of different units of power, according to Section 4.2 . The three levels of flexibility introduced in that section are also considered here through a sensitivity analysis.

The requested load reduction is distributed among all the consumer groups (with only slight differences between the different degrees of flexibility), as illustrated in Fig. 3a This is because the aggregator uses the least critical part of the demand of each consumer group to achieve the requested load reduction. Therefore, the total inconvenience cost is significantly reduced (more than 90\%) with respect to scenario 1, as illustrated in Fig. 3b. This reduction is enhanced as we move from a case with low flexibility to a case with high flexibility as the proportion of load that the consumers perceive as non-critical is increased.

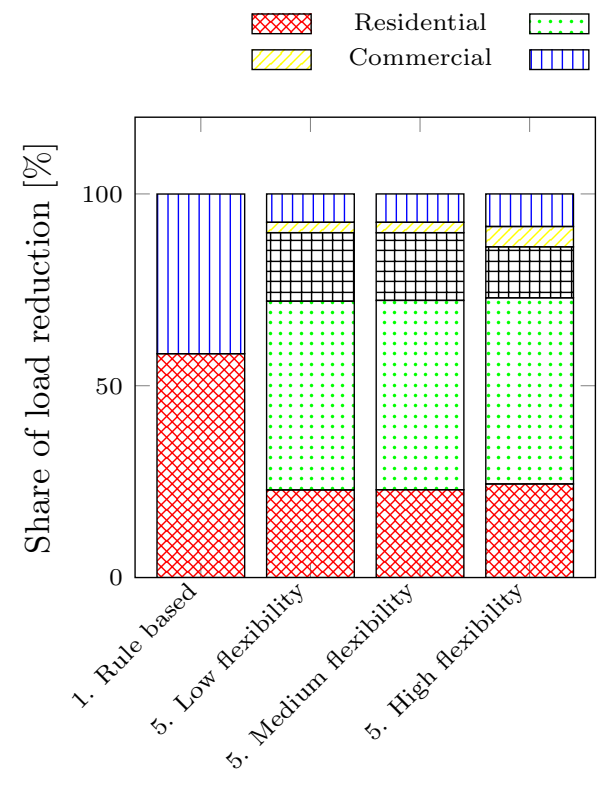

(a) Distribution of load reduction
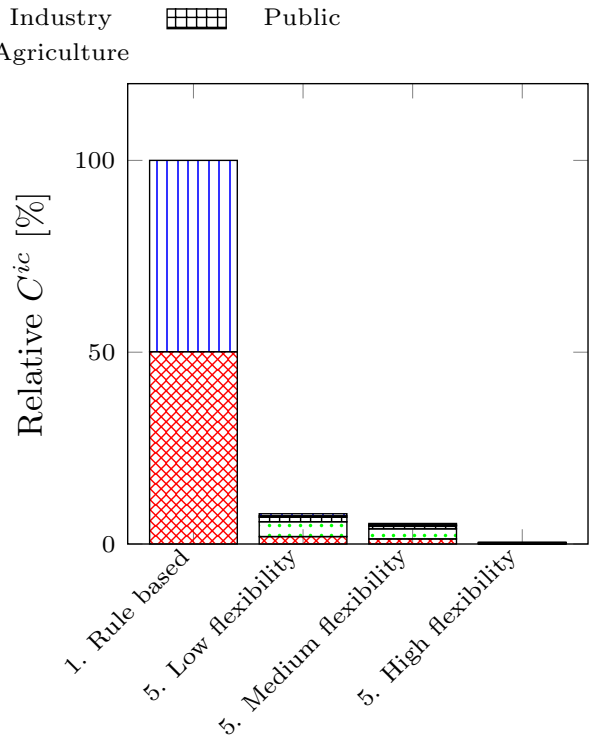

(b) Relative total inconvenience cost (with respect to rule-based approach)

Figure 3: Distribution of load reduction (a) and total inconvenience cost (b) over the aggregator's portfolio in scenario 5. 


\subsection{Scenario 6: Temporal redistribution of shiftable loads}

While scenarios 1-5 assume that consumers' power can be either supplied or curtailed, scenario 6 accounts for the possibility of temporally redistributing a part of the load that corresponds to shiftable

appliances by delaying the activation of their cycles. As discussed in Section 5.2, only residential consumers are assumed to have shiftable loads in the examined case study. Furthermore, in this study we assume that the residential consumers have indifferent activation preferences regarding the operation of their shiftable appliances (Section 4.3 . Eq. (35)). Different cases regarding the maximum activation delay of these appliances are considered 5

As the maximum delay is increased, a higher share of the requested load reduction is satisfied through the temporal redistribution of shiftable loads, as illustrated in Fig. 4a. Furthermore, the total inconvenience cost is reduced since the inconvenience associated with shifting load is generally lower than the inconvenience associated with curtailing load (Section 4.1 and 4.3), as illustrated in Fig. 4b. These effects of load shifting cannot be captured by state-of-the-art, static models, which do not encapsulate the time-coupling characteristics of shiftable loads.
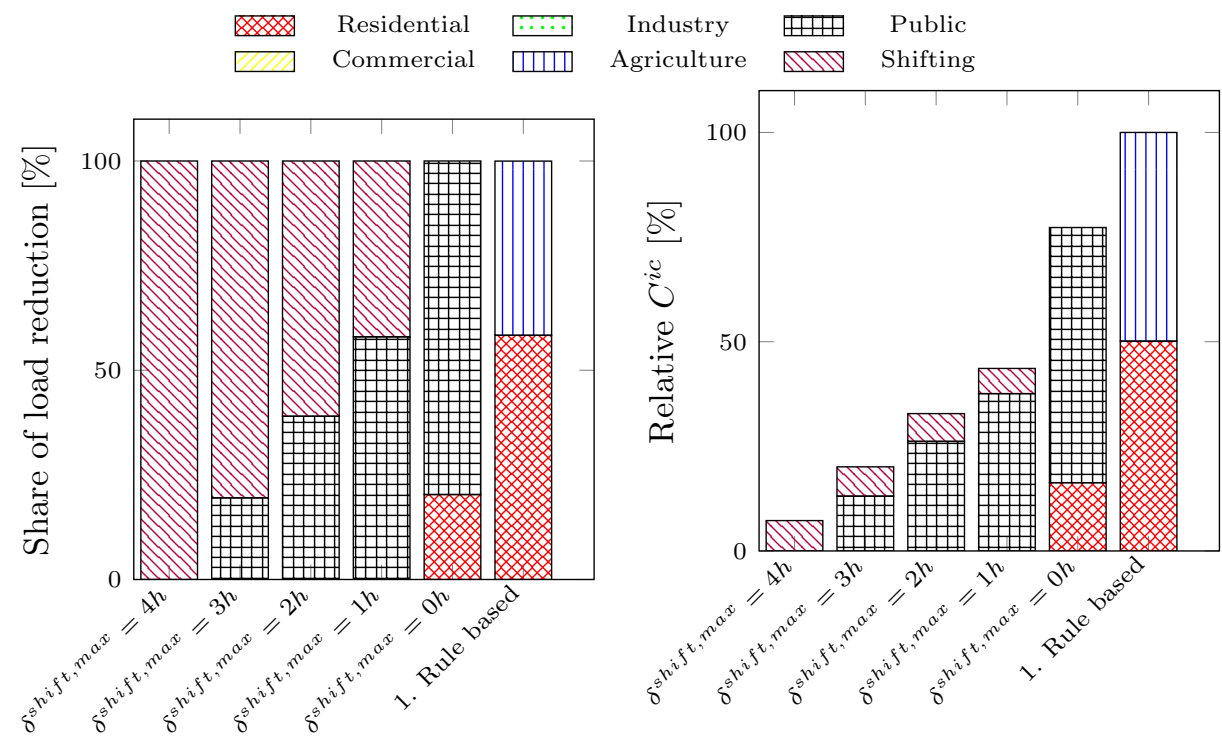

(a) Distribution of load reduction

(b) Relative total inconvenience cost (with respect to rule-based approach)

Figure 4: Distribution of load reduction (a) and total inconvenience cost (b) over the aggregator's portfolio in scenario 6.

\footnotetext{
${ }^{5}$ Scenario 6 assumes that each consumer values all its units of power of curtailable load equally.
} 


\subsection{Implementation and computational requirements}

The developed model has been coded in the Julia programming language 29] using the Jump Package [30] and solved using the Mosek Mixed Integer Linear Program branch and bound solver 31] on a computer with an Intel(R) Core(TM) i7-6600U CPU @2.60GHz 2.80GHz processor and 16 GB of RAM. The optimization problem corresponding to the above case study with 30 consumers consists of 750 and 1210 continuous variables and 570 and 830 integer variables for scenarios 5 and 6 , respectively, which constitute the most complex ones in terms of modelling detail. This problem required less than 0.01 second to be solved in all the examined scenarios.

In order to further investigate the computational requirements and scalability of the proposed model, we have carried out additional case studies with an increasing number of consumers in the aggregator's portfolio, reaching in the extreme case 30,000 consumers. In each of these cases, the total load reduction requested from the aggregator at each hour of the adequacy issue is proportionally increased, while the relative distribution of the consumers over different types and slices follows the one outlined in Table 5 .

Figure 5 and Table 8 present the computational time requirements and the number of integer variables of the optimization problem corresponding to each of the examined cases, for scenarios 5 and 6 . The most significant finding is that the proposed model scales very satisfactorily with the number of consumers, and the highest recorded computational time (corresponding to scenario 6 in the case with 30,000 consumers) is lower than 10 seconds, demonstrating the practical applicability of the model. Scenario 6 involves a significantly higher number of integer variables and consequently higher computational time requirements for the same number of consumers, with respect to scenario 5 (and the rest of the examined scenarios), due to the consideration of shiftable loads (Section 3.2).

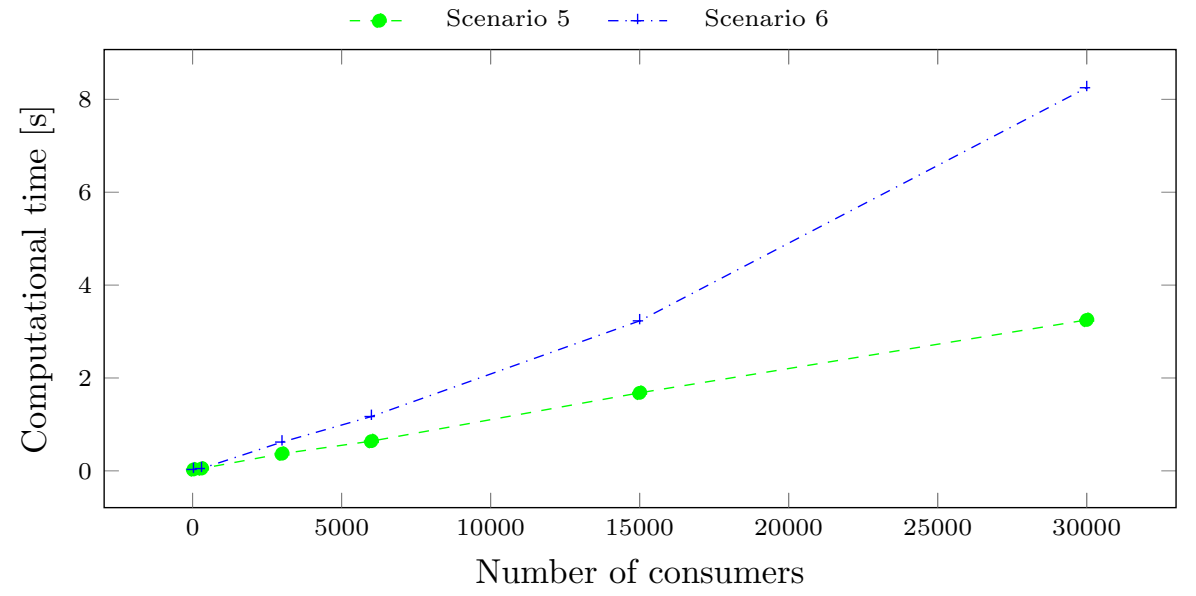

Figure 5: Computational time requirements of the proposed model for different numbers of consumers in the aggregator's portfolio. 
Table 8: Number of integer variables of the proposed model for different numbers of consumers in the aggregator's portfolio.

\begin{tabular}{c|cccccc} 
& \multicolumn{6}{|c}{ Number of consumers } \\
& 30 & 300 & 3000 & 6000 & 15000 & 30000 \\
\hline Scenario 5 & 570 & 5700 & 57000 & 114000 & 285000 & 570000 \\
Scenario 6 & 830 & 8300 & 83000 & 166000 & 415000 & 830000
\end{tabular}

\subsection{Fairness of load management decisions} and 6) the proposed model enhances the equity of the load management decisions. At the same time, these decisions are not aligned with the principle of equality, since different consumers are treated in a different way. Overall, and although we recognize that the above analysis of fairness principles is not 

offers clear benefits in terms of economic efficiency (Sections 5.35.8), practical applicability (Section 5.9 and equity.

\section{Conclusion and future work}

Despite the significant potential of demand flexibility in supporting the secure and cost-efficient operation of the power system, the consumers' inconvenience associated with the modification of their electricity demand patterns has not been comprehensively modelled. This paper has proposed a novel dynamic model of the inconvenience cost which realistically captures a number of relevant factors, including differentiated preferences of different consumer groups, the time and duration of interruptions, the differentiated valuation of different units of power and the temporal redistribution of shiftable loads.

The proposed model has been applied in the examined case studies to the load portfolio management of an aggregator in a scenario involving emergence of an adequacy issue in the Belgian system. The results demonstrate how the consideration of each of the above factors affects the distribution of the requested load reduction among different consumer groups and the total inconvenience cost, revealing the value of the proposed model.

Considering these results, a clear direction for future work lies in collecting additional and more detailed information regarding the consumers' inconvenience perceptions, through suitably designed surveys as well as automated data collection approaches enabled by emerging advanced metering and communication technologies. First of all, surveys quantifying the impact of differentiated preferences of different consumers groups and the time and duration of interruptions have been carried out only in a limited number of countries, such as the case of Norway of which the outcomes have been employed in this paper ${ }^{6}$ Going further, to the best of the authors' knowledge, no surveys quantitatively characterising the differentiated valuation of different units of power and the temporal redistribution of shiftable loads have been carried out so far; the presented results indicate that the consideration of these factors in the representation of consumers' inconvenience can potentially yield significant reductions of the total inconvenience cost, since the least critical part of each consumer's demand can be curtailed when required and the inconvenience cost of load shifting is generally lower than the inconvenience cost of load curtailment.

Finally, it should be noted that beyond the application of the proposed model in the presented case studies (aggregator's portfolio management during adequacy issues), the authors envisage that future work will apply this model to a number of more complex applications involving the deployment

\footnotetext{
${ }^{6}$ The Fourth Energy Package of the European Commission prescribes that all member states have to establish at least a single estimate of VOLL for their territory and can establish a VOLL per bidding zone, if they have several ones.
} 
of demand flexibility. These may include the formation of efficient bidding strategies by aggregators in energy and balancing markets, the design of efficient pricing tariffs and incentive schemes by electricity suppliers, and the incorporation of the impact of demand flexibility in generation and network investment decisions.

\section{Acknowledgment}

The research of Evelyn Heylen has been funded by a PhD Fellowship and a travel grant of the Research Foundation Flanders (FWO), Belgium. This research is partly funded by the European Horizon 2020 project EU Sysflex under grant agreement no. 773505.

\section{Bibliography}

[1] M. Amin, Toward self-healing energy infrastructure systems, IEEE Computer Applications in Power 14 (1) (2001) 20-28.

[2] U.S.-Canada Power System Outage Task Force, Final Report on the August 14, 2003 Blackout in the United States and Canada: Causes and Recommendations, Tech. rep. (2014).

[3] E. Heylen, G. Deconinck, D. Van Hertem, Impact of increased uncertainty in power systems on performance of short term reliability management, in: International Conference on Probabilistic Methods Applied to Power Systems, 2016.

[4] D. Papadaskalopoulos, R. Moreira, G. Strbac, D. Pudjianto, P. Djapic, F. Teng, M. Papapetrou, Quantifying the Potential Economic Benefits of Flexible Industrial Demand in the European Power System, IEEE Transactions on Industrial Informatics 14 (11) (2018) 5123 - 5132.

[5] Energiepact: Het energieoverleg tussen de federale staat en de gewesten: Resultaten burgerbevraging energiepact [In Dutch], [Online] https://www.energiepact2050.be/ 129-17-brochure-A4-N.pdf [Accessed 05/09/2018] (2017).

[6] M. Ovaere, E. Heylen, S. Proost, G. Deconinck, D. Van Hertem, How detailed value of lost load data impact power system reliability decisions, Energy Policy 132 (2019) 1064-1075.

[7] A.-H. Mohsenian-Rad, V. W. Wong, J. Jatskevich, R. Schober, A. Leon-Garcia, Autonomous demand-side management based on game-theoretic energy consumption scheduling for the future smart grid, IEEE transactions on Smart Grid 1 (3) (2010) 320-331.

[8] Y. Liu, C. Yuen, S. Huang, N. U. Hassan, X. Wang, S. Xie, Peak-to-average ratio constrained demand-side management with consumer's preference in residential smart grid, IEEE Journal of Selected Topics in Signal Processing 8 (6) (2014) 1084-1097. 
[9] C. O. Adika, L. Wang, Autonomous appliance scheduling for household energy management, IEEE transactions on smart grid 5 (2) (2014) 673-682.

[10] F.-L. Meng, X.-J. Zeng, A stackelberg game-theoretic approach to optimal real-time pricing for the smart grid, Soft Computing 17 (12) (2013) 2365-2380.

[11] A. J. Conejo, J. M. Morales, L. Baringo, Real-Time Demand Response Model, IEEE Transactions on Smart Grid 1 (3) (2010) 236-242.

[12] G. K. Toh, H. B. Gooi, Procurement of interruptible load services in electricity supply systems, Applied Energy 98 (2012) 533-539.

[13] H. Karimi, D. Papadaskalopoulos, G. Strbac, Integrating wet appliances with delay functionality in distribution network operation and planning, CIRED-Open Access Proceedings Journal 2017 (1) (2017) 1836-1840.

[14] F. Meng, X. Zeng, A Profit Maximization Approach to Demand Response Management with Customers Behavior Learning in Smart Grid, IEEE Transactions on Smart Grid 7 (3) (2016) $1516-1529$.

[15] C. Chen, S. Kishore, L. V. Snyder, An innovative rtp-based residential power scheduling scheme for smart grids, in: Acoustics, Speech and Signal Processing (ICASSP), 2011 IEEE International Conference on, IEEE, 2011, pp. 5956-5959.

[16] H. Karimi, D. Papadaskalopoulos, G. Strbac, Integrating customers' differentiated supply valuation in distribution network planning and charging, in: European Energy Market (EEM), 2016 13th International Conference on the, IEEE, 2016, pp. 1-5.

[17] S. Junlakarn, M. Ilic, Distribution System Reliability Options and Utility Liability, IEEE Transactions on Smart Grid 5 (5) (2014) 2227-2234.

[18] M. Fahrioglu, F. L. Alvarado, Using utility information to calibrate customer demand management behavior models, IEEE Transactions on Power Systems 16 (2) (2001) 317-322.

[19] W. Powel, Approximate dynamic programming: Solving the curses of dimensionality, Wiley, Hoboken, New Jersey, 2011.

[20] OECD, Willingness to Pay vs. Willingness to Accept, in: Cost-Benefit Analysis and the Environment, OECD Publishing, 2006, pp. 155-167.

[21] GARPUR consortium, D3.1 Quantification method in the absence of market response and with market response taken into account, [Online] http://www.garpur-project.eu/deliverables [Accessed 19/11/2018] (2016). 
[22] EnergiNorge, Samfunns $\varnothing$ konomiske Kostnader Ved Avbrudd Og Spenningsforstyrrelser, no. 349, [In Norwegian], 2012.

[23] M. Hofmann, H. Seljeseth, G. Holst Volden, G. Kjølle, Study on estimation of costs due to electricity interruptions and voltage disturbances, Tech. rep., Sintef (2010).

[24] G. Kjølle, K. Samdal, K. Brekke, Incorporating short interruptions and time dependency of interruption costs in continuity of supply regulation, in: CIRED 2009 - 20th International Conference and Exhibition on Electricity Distribution - Part 1, 2009, pp. 1-4.

[25] H. Karimi Khouzani, Integrating consumers supply valuation and flexibility in distribution network planning and charging, Ph.D. thesis, Imperial College London, UK (Dec. 2016).

[26] H. P. Williams, Logic and Integer programming, Vol. 130, Berlin: Springer, 2009.

[27] FICO Xpress Optimization Suite, MIP formulations and linearization: Quick reference, Tech. rep., FICO (2009).

[30] I. Dunning, J. Huchette, M. Lubin, Jump: A modeling language for mathematical optimization, SIAM Review 59 (2) (2017) 295-320.

[31] The mosek optimization software, [Online] https://www.mosek.com/ [Accessed 13/02/2019].

[32] J. Konow, Which is the fairest one of all? A positive analysis of justice theories, Journal of

[33] E. Heylen, M. Ovaere, S. Proost, G. Deconinck, D. Van Hertem, Fairness and inequality in power system reliability: Summarizing indices, Electric Power Systems Research 168 (3) (2019) 313-323. 